\title{
CONTRAST-INDUCED NEPHROPATHY (CIN) IN 118 CORONARY ANGIOGRAPHIC CASES
}

\author{
AFMSHAMSUL HAQUE ${ }^{1}$, MD GOLAM RABBANI, MUHAMMAD RABIUL HOSSAIN, MD. HAYDER \\ RASHID, MAMUN MOSTAFI, A K M MIJANUR RAHMAN
}

\begin{abstract}
Contrast-induced nephropathy (CIN) is the third leading cause of hospital-acquired renal failure and is associated with significant morbidity and mortality. The aim of the present study was to find out the incidence and outcome of CIN in patients undergoing coronary angiography using standard cardiac cath lab protocol. This observational study was carried out in patients undergoing Coronary angiography with or without Percutaneous coronary interventions (PCI) in CMH Dhaka from July 2007 to July 2008. Exclusion criteria were chronic kidney disease (CKD), nephrotoxic medications, severe hypotension, severe heart failure (NYHA class IV) and severe anaemia. Out of 118 cases two demonstrated impaired renal function and both of them were diabetic. The incidence was found to be $1.69 \%$ and outcome was satisfactory in these cases. Renal function of both the patients gradually became normal within next two weeks. No patient required dialysis.
\end{abstract}

\section{Introduction}

CIN is one of the common causes of acute renal insufficiency after cardiovascular procedures. Acute renal failure in connection with the administration of intravascular iodine-based contrast media $(\mathrm{CM})$ was recognized long ago. CIN is defined as an impairment of renal functions subsequent to the administration of $\mathrm{CM}$ in the absence of any cause. CIN is diagnosed when there is an increase in serum creatinine concentration of e" $0.5 \mathrm{mg} / \mathrm{dl}$ or relative increase of e" $25 \%$ from the baseline within $72 \mathrm{hrs}$ after CM administration. ${ }^{1}$ The recovery occurs in majority of cases within 2-3 weeks.

Several risk factors for CIN have been defined, chronic renal insufficiency being the most important particularly in connection with diabetes mellitus. ${ }^{2,3}$ Others are DM, nephrotoxic medications (e.g. NSAID, ACE inhibitors, Cyclosporine, amphotericin B), severe hypotension, heart failure, severe anaemia and age > 75 years. The incidence of CIN in general population is low and reported to be 0.6 to $2.3 \% .^{4}$ It is also observed that CIN is rare if the renal function is normal. CIN is usually reversible and begins soon after CM is administered. The variation of reported incidence of CIN depends on patient selection, type of procedure, osmolarity and amount of CM used, prophylactic measures and definition of CIN.
Identification of risk factors, prophylactic measures and the measures to reduce the offending drug may be useful to decrease the risk of CIN. Our aim with the present study was to find out the incidence and outcome of CIN in patients undergoing CAG procedures using standard cardiac cath lab protocol.

\section{Materials and Methods}

An observational study was done on the incidence of Contrast-induced nephropathy (CIN) in patients undergoing Coronary angiography in $\mathrm{CMH}$ Dhaka from July 2007 to July 2008. 118 Coronary angiographies with or without percutaneous coronary intervention $(n=118)$ performed in this hospital were evaluated. All patients received tri-iodinated non-ionic water-soluble contrast medium (Diapamiro 370 Iopamidol at the concentration of $370 \mathrm{mg}$ of iodine / $\mathrm{ml}$ ) during coronary angiography. Out of 118 cases, PCI was done in 36 cases. A total of 130 patients were initially selected who were found to have a preangiographic serum-creatinine ( $\mathrm{s}-\mathrm{Cr})$ of $1.4 \mathrm{mg} / \mathrm{dl}$ or less. Postprocedural serial s-Cr values were followed up in 118 patients and these patients were included in the study. After the procedure s-Cr was measured at $24 \mathrm{hrs}, 72 \mathrm{hrs}$ and after 14 days. If abnormal s-Cr was found, cases were followed up further till normal creatinine level. Out of these patients 20 patients were diabetic, 46 patients were hypertensive. Exclusion

1. Classified Specialist in Medicine and Cardiologist, CMH Dhaka, Dhaka Cantonment, Dhaka, Bangladesh. Correspondence : Lt Col (Dr.) A F M Shamsul Haque, Classified Specialist in Medicine and Cardiologist, CMH Dhaka, Dhaka Cantonment, Dhaka, Bangladesh. 
criteria were Chronic kidney disease (CKD), nephrotoxic medications, severe hypotension, severe heart failure (NYHA class IV) and severe anaemia.

\section{Results}

Amongst patients receiving low osmolar contrast media (CM), two patients demonstrated more than $25 \%$ rise in s-Cr. Both the patients had rise of s- $\mathrm{Cr}$ after $24 \mathrm{hrs}$ of CAG and maximum rise was 1.9 to 2.0 $\mathrm{mg} / \mathrm{dl}$ and gradually became normal within next two weeks. After exclusion of other explanations of impaired renal function these two patients (1.69\%) were considered to have developed CIN. Both the patients were diabetic. Both of them regained normal renal function completely. No patient required dialysis.

Table-I

Demographic characteristics $(n=118)$

\begin{tabular}{lc}
\hline Age & Number $(\%)$ \\
\hline$<40$ Yrs & $18(15.25)^{*}$ \\
$41-50$ Yrs & $38(32.20)$ \\
$>50$ Yrs & $62(52.54)$ \\
\hline
\end{tabular}

*Figures in the parentheses indicate corresponding \%

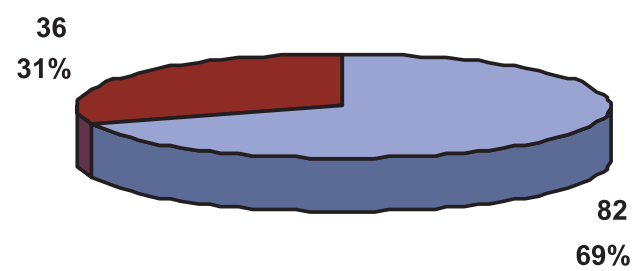

Fig. 1: Sexdistribution $(n=118)($ Male $=82$, Female $=36)$

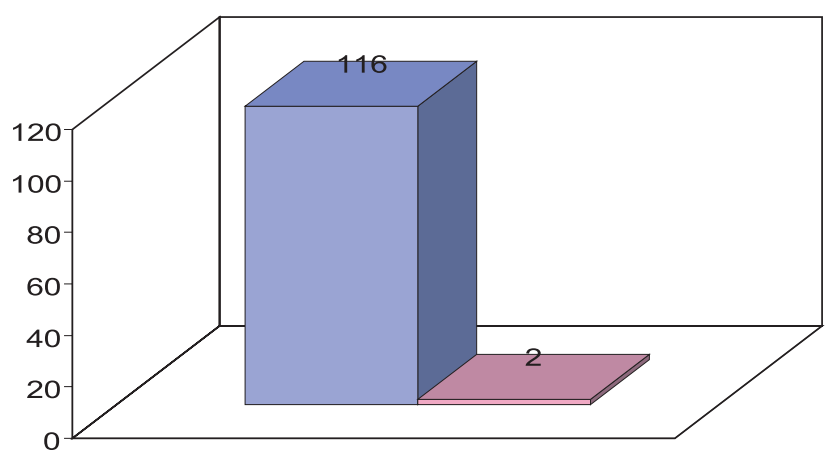

Fig.-2: Number of cases with CIN $(n=2)$

\section{Discussion}

CIN is a cause of acute renal deterioration, occurring after arterial and venous $\mathrm{CM}$ administration. The single most important risk factor for CIN is preexisting renal failure and diabetes mellitus is the second most important risk factor. Drugs including NSAID, cyclosporine and aminoglycosides can potentiate the nephrotoxic effect if given with iodinated contrast media. Some authors consider more than $25 \%$ increase of serum-creatinine above base-line level to define CIN. Risk factors are primarily renal insufficiency and diabetic nephropathy, but conditions like old age, congestive cardiac failure and dehydration can influence the outcome. The pathogenesis of CIN is complex. The underlying pathophysiological mechanism may include direct tubular toxicity and haemodynamic effects. ${ }^{5}$ Although CIN is generally limited to a transient decline in renal function, it is not a benign complication in high risk persons. Radio contrast exposure is associated with vasoconstriction of the renal vascular bed and, in certain circumstances, with acute renal failure. This may be influenced by the volume of contrast infused or underlying disease, such as diabetes or renal failure. The cornerstone of CIN prevention, in both the high and low risk patients, is adequate parenteral volume repletion. Important measures should be taken primarily by the interventionist, i.e. reducing the $\mathrm{CM}$ dose by applying meticulous angiographic technique suitable for each patient. Knowledge about the influence of CM on kidney function has increased as has information concerning prophylactic measures. ${ }^{6}$ Recognition of the high risk patient coupled with appropriate peri-procedural management can reduce the incidence of CIN. An interval s-Cr check-up should be done before repeated CM procedures or major surgery is done. The important step to prevent CIN is proper hydration instituted before, during and after the administration of CM. ${ }^{7}$ Current evidence says that low-osmolar CM has been shown to be less nephrotoxic than high-osmolar agents in risk patients. ${ }^{2}$

Our observational study focused on patients of angiographic examinations including interventional therapeutic procedures performed with low-osmolar CM intended to find out the incidence and outcome of CIN. The incidence was found to be $1.69 \%$ and outcome was satisfactory in these cases. Lasser and Lyon found in their study that the incidence of CIN in general population is low and reported to be 0.6 to $2.3 \% .{ }^{4}$ Our finding is also similar. Po-Tsang, Kang-Ju and Hua- 
Chang have mentioned that the incidence of CIN in general population is estimated at $1 \%$ to $2 \%$ which is similar to our finding. ${ }^{9}$

\section{Conclusion}

Although CIN is usually self-limited, it may cause permanent renal injury and even lead to dialysis in patients with pre-existing renal impairment. Development of CIN may result in increased inhospital complications, morbidity and mortality. In patients with preangiographic normal renal function, the prevalence is low but in pre-existing renal impairment it may pose a serious threat. Adequate prophylactic measures can reduce the morbidity and mortality. The present observational study indicates that the risk of CIN in connection with coronary angiography is low when modern low-osmolar CM is used.

\section{References}

1. Gupta S, Gupta MM. Prevention of Contrast Induced Nephrology-An Update.Indian Heart J 2007; 59(4): 375-377.

2. Rudnick MR, Goldfarb S, Wexler L, et al. Nephrotoxicity of ionic and nonionic contrast media in 1196 patients. A randomized trial. Kidney Int 1995; 47: $254-61$.
3. Morcos SK. Contrast medium-induced nephrotoxicity. In: Dawson P, Cosgrove DO, Grainger RG, eds. Textbook of Contrast Media. Oxford: Isis Medical Media Ltd, 1999:135-48.

4. Lasser EC, Lyon SG, Berry CC. Reports on Contrast media reactions: analysis of data from reports to the US Food and Drug Administration. Radiology 1997; 203: 605-610.

5. G Sternal, U. Nyman and T Valdes, Low risk of contrast-medium-induced nephropathy with modern angiographic technique. J Intern Med 2001; 250: $429-434$

6. Kolonko A, Kokot F, Wiecek A. Contrast-associated nephropathy-old clinical problem and new therapeutic perspectives. Nephrol Dial Transpl 1998; 13: 803-6.

7. Wideroe TE, Tetsche K, Damgaard-Mörch P, et al. Cellular and renal toxicity of a urographic contrast medium used in high concentration in uremic patients. Scand J Urol Nephrol 1980; 14: 101-5.

8. Lee PT, Chou KJ, Fang HC. Preventing contrastinduced nephropathy in patients with baseline renal dysfunction undergoing coronary angiography. Curr Treat Options Cardiovasc Med 2009;11:71-78. 